\title{
Investigation of a severe SARS-CoV-2 outbreak in a long-term care home early in the pandemic
}

\author{
Michelle Murti MD MPH, Monika Goetz MSc, Andrea Saunders RN MSc, Vidya Sunil DVM MSc, Jennifer L. Guthrie PhD, \\ AliReza Eshaghi PhD, Sandra Zittermann PhD, Sarah Teatero MSc, Nahuel Fittipaldi PhD, Heather Rilkoff MPH, \\ Jonathan B. Gubbay MBBS MMedSc, Gary Garber MD, Sandra Callery RN MHSc, Anne Marie Holt MHSc,
} A. Lynn Noseworthy MD MHSc

Cite as: CMAJ 2021 May 10;193:E681-8. doi: 10.1503/cmaj.202485

\begin{abstract}
BACKGROUND: The implementation of outbreak management measures has decreased the frequency and severity of SARS-CoV-2 outbreaks in Ontario longterm care homes. We describe the epidemiological and laboratory data from one of the first such outbreaks in Ontario to assess factors associated with its severity, and the impact of progressive interventions for infection control over the course of the outbreak.
\end{abstract}

METHODS: We obtained line list and outbreak data from the public health unit to describe resident and staff cases, severity and distribution of cases over time and within the outbreak facility.
Where available, we obtained data on laboratory specimens from the Public Health Ontario Laboratory and performed whole genome sequencing and phylogenetic analysis of viral specimens from the outbreak.

RESULTS: Among 65 residents of the long-term care home, 61 (94\%) contracted SARS-CoV-2, with a case fatality rate of $45 \%$ (28/61). Among 67 initial staff, 34 (51\%) contracted the virus and none died. When the outbreak was declared, 12 staff, 2 visitors and 9 residents had symptoms. Resident cases were located in 3 of 4 areas of the home. Phylogenetic analysis showed tight clustering of cases, with only 1 additional strain of genetically distinct SARS-CoV-2 identified from a staff case in the third week of the outbreak. No cases were identified among 26 new staff brought into the home after full outbreak measures were implemented.

INTERPRETATION: Rapid and undetected viral spread in a long-term care home led to high rates of infection among residents and staff. Progressive implementation of outbreak measures after the peak of cases prevented subsequent staff cases and are now part of long-term care outbreak policy in Ontario.

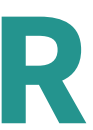

esidents of long-term care (LTC) homes have been disproportionately represented in overall cases of SARSCoV-2 and COVID-19 both in Canada and internationally. ${ }^{1-3}$ Older adults in LTC are vulnerable to SARS-CoV-2 and are at increased risk of complications and death given their underlying health status and age. ${ }^{4}$ In Ontario, LTC residents accounted for $18 \%$ of confirmed cases during the first wave of the pandemic that ended by June 2020. As of Feb. 6, 2021, LTC residents accounted for $5.3 \%$ of confirmed cases of COVID-19 and had an overall case fatality rate of $25.4 \%$, compared with $2.4 \%$ for all cases in Ontario. ${ }^{5}$

In Ontario, LTC homes were provided provincial direction on enhanced measures to prevent COVID-19 as of Mar. 9, 2020; updated directives were provided over time to include measures such as expanded testing indications, visitor policies, staff work locations, universal masking and strict outbreak definition and management. ${ }^{6,7}$ Implementation of these measures corresponded with fewer and less severe outbreaks over the course of the pandemic's first wave in Ontario. ${ }^{8}$

We report on the epidemiological data, infection prevention and control (IPAC), laboratory testing and phylogenetic analysis of one of the earliest and largest COVID-19 outbreaks in an Ontario LTC home, and the impacts of progressive outbreak management measures that eventually influenced provincial guidance for LTC outbreak management.

\section{Methods}

Sources of data and epidemiological analysis Outbreaks of respiratory infections in LTC and cases of COVID-19 are reportable to public health in Ontario under the Health Protection and Promotion Act. ${ }^{9}$ Using data extracted from the province's integrated Public Health Information System (iPHIS) on June 11, 2020, we analyzed COVID-19 cases among staff, 
residents and close contacts of residents (visitors) that were linked to this outbreak. ${ }^{10}$ We extracted case data on age, sex, date of symptom onset, symptoms, specimen collection date and laboratory testing history.

We categorized confirmed and probable cases according to the provincial case definition at the time, where confirmed cases had laboratory confirmation of SARS-CoV-2. Probable cases were defined as those with 2 or more symptoms (abnormal temperature, lethargy, nasal congestion, new or worsening cough, loss of appetite or hoarseness, difficulty swallowing or sore throat) with an onset date of Mar. 9, 2020, or later, and either no laboratory testing or an inconclusive result. ${ }^{11}$ We obtained lists of staff and residents at the onset of the outbreak for initial at-risk denominators. No additional residents were admitted during the outbreak; additional staff were brought in for support throughout the outbreak. We obtained information on implemented IPAC measures from the public health unit outbreak management summary. We mapped residents by room occupancy at the start of the outbreak, with progression of confirmed and probable cases by single, double and quadruple rooms.

\section{Laboratory analysis}

Laboratory testing was initially conducted primarily at the Public Health Ontario Laboratory, with later samples tested across the Ontario laboratory network. Public Health Ontario Laboratory testing used either a laboratory-developed test targeting the SARSCoV-2 envelope (E) gene, or a commercial assay targeting the $E$ and orf1ab genes (cobas SARS-CoV-2 test, Roche Diagnostics). ${ }^{12}$

We conducted whole genome sequencing of SARS-CoV-2 specimens with sufficient viral quantities at the Public Health Ontario Laboratory (for technical details, please see Appendix 1, available at www.cmaj.ca/lookup/doi/10.1503/cmaj.202485/tab -related-content). We uploaded consensus sequences to GISAID (www.gisaid.org/) and accession numbers are available in Appendix 2 at www.cmaj.ca/lookup/doi/10.1503/cmaj.202485/ tab-related-content.

\section{Statistical analysis}

For epidemiological data, we report the attack rate of confirmed and probable cases of COVID-19 among residents and staff, representing the percentage of the at-risk population who became infected with SARS-CoV-2 during the outbreak.

For laboratory data, we reviewed positive specimens by the cycle threshold value of the $E$ gene from the laboratorydeveloped test (indeterminate 38.1-39.9, negative $\geq 40$ ) and the cobas test from Roche (cycle threshold value interpreted as a negative result not specified by manufacturer). Cycle threshold values represent the number of polymerase chain reaction amplification cycles required to detect the target, with higher values corresponding to lower amounts of virus in the specimen. We calculated independent Student $t$ tests using SAS Enterprise Guide version 7.1 to compare the mean cycle threshold values of initial specimens from asymptomatic and symptomatic cases and to compare any specimen collected $\leq 14$ days with those collected $>14$ days after symptom onset (for symptomatic cases).

\section{Ethics approval}

The Public Health Ontario Ethics Review Board determined that this project did not require research ethics committee approval as the activities described in this manuscript were conducted in fulfillment of Public Health Ontario's legislated mandate "to provide scientific and technical advice and support to the health care system and the Government of Ontario in order to protect and promote the health of Ontarians" (Ontario Agency for Health Protection and Promotion Act, SO 2007, c 10) and are therefore considered public health practice, not research.

\section{Results}

A cluster of residents with acute respiratory illness was initially identified in an LTC home on Mar. 17, 2020, with 3 testing positive for SARS-CoV-2 on Mar. 20. Initial outbreak measures in accordance with 2018 respiratory outbreak guidance were initiated the evening of Mar. 17; the outbreak was declared on Mar. 18, based on the provincial case definition of respiratory outbreaks, in the absence of specific guidance and definitions for COVID-19 (Figure 1). ${ }^{9,13}$ Additional measures specific for COVID-19 were implemented Mar. 20, including placing all residents on droplet and contact precautions and universal masking of staff. All LTC homes in Ontario had been closed to visitors as of Mar. 14 , by provincial directive. ${ }^{11}$

Between Mar. 20 and Mar. 26, specimens from 28 staff and 2 visitors to the home tested positive for SARS-CoV-2. On case interview, 12 staff, 2 visitors and 9 residents reported symptom onset between Mar. 9 and Mar. 18. Between Apr. 1 and Apr. 3, the home began testing of all newly deceased residents and symptomatic residents, including those who had developed symptoms since the start of the outbreak, identifying 27 additional residents positive for SARS-CoV-2. On Apr. 6, testing of asymptomatic residents was initiated (Figure 1). The last case identified among residents had a positive specimen collected Apr. 10 with symptom onset Apr. 30. The last case identified among staff had symptom onset on Apr. 20. The outbreak was declared over on May 14, 2020.

At the start of the outbreak, 65 residents and 67 staff were associated with the LTC home. Including confirmed and probable cases, there was a resident attack rate of $94 \%(n=61)$ and a staff attack rate of $51 \%(n=34)$. Resident and staff cases are described in Table 1 . There were no asymptomatic cases reported for staff; among resident cases, 5\% $(n=3)$ developed symptoms after collected specimens tested positive (presymptomatic) and $13 \%(n=8)$ remained asymptomatic (including 7 confirmed asymptomatic cases and 1 probable asymptomatic case in a resident with no documented COVID-19 symptoms whose death was attributed to SARS-CoV-2 after assessment by the public health unit). Most symptomatic residents and staff reported cough and fever or abnormal temperature. For staff, headache was the most common symptom. No residents or staff were hospitalized for COVID-19. Of note, most residents had donot-resuscitate orders $(n=60)$. The case fatality rate was $46 \%$ $(n=28)$ for the 61 residents with confirmed and probable SARSCoV-2 infections; no staff deaths were reported. Both visitors required hospitalization for COVID-19 and 1 visitor died. 


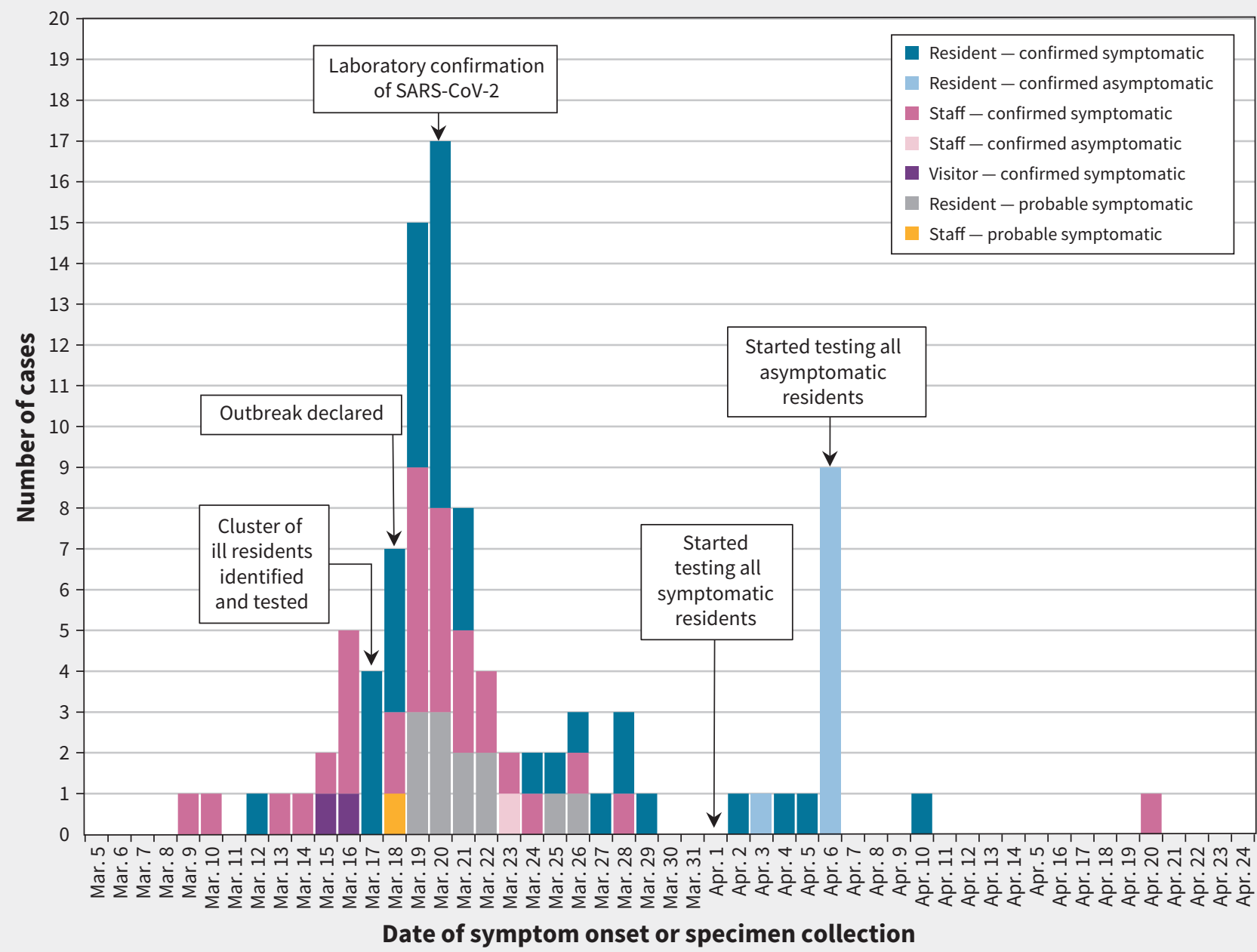

Figure 1: Number of confirmed and probable cases of COVID-19 among residents, staff and visitors $(n=96)$ associated with an outbreak in a long-term care home, by date of symptom onset date or specimen collection (if asymptomatic) from March and April 2020. Figure excludes 1 probable case in a resident who did not have symptoms recorded before death and was not tested, but that was determined to be a probable case based on assessment by the public health unit.

Residents with symptom onset before the outbreak declaration were in 3 out of 4 resident blocks of the home, and in a mix of single, double and quadruple occupancy rooms (Table 2). The index resident case was in a quadruple room. By Mar. 21, after the peak of resident cases, all areas of the home were affected.

Early in the outbreak, there were critical staffing shortages because of the high and rapid staff attack rate. Additional staff $(n=26)$ were introduced over the course of the outbreak. To our knowledge, all staff cases occurred among the original 67 staff, with no additional cases reported among subsequent staff, giving a final staff attack rate of $37 \%(n=34)$ among all 93 staff members. Preexisting staffing shortages led to staff working while symptomatic before the outbreak was declared (Mar. 18). Some staff were allowed to work while masked once their symptoms improved, caring for residents who had already tested positive for SARSCoV-2, while still self-isolating at home. Although IPAC measures specific for COVID-19 were implemented on Mar. 20, including droplet and contact precautions for all residents (symptomatic or not), critical shortages in personal protective equipment early in the outbreak resulted in its underuse and reuse by staff.

We obtained cycle threshold values for 79 initial and 31 follow-up specimens. Of the 79 initial specimens, 69 were from symptomatic cases and 10 were from asymptomatic cases. Mean cycle threshold values for the $\mathrm{E}$ gene were significantly lower (i.e., viral loads were higher) in initial specimens collected from symptomatic cases $(24.7,95 \%$ confidence interval $[\mathrm{Cl}]$ 22.9-26.5) compared with asymptomatic cases (33.3; 95\% Cl 31.0-35.6, $p<0.001$ ). Cycle threshold values were also significantly lower among the 60 specimens collected from symptomatic cases within 14 days of symptom onset $(23.6,95 \% \mathrm{Cl} 21.8-$ 25.5) compared with the 33 specimens collected 14 days after symptom onset (35.4, 95\% Cl 34.4-36.4, $p<0.001$ ).

Specimens from 24 staff and 4 residents were available and had sufficient viral quantities for genome sequencing. The average genome coverage was $99.6 \%$. Overall, outbreak specimens had 6-9 single nucleotide polymorphisms (SNPs) 
Table 1: Demographic characteristics, symptoms and outcome severity of confirmed and probable COVID-19 among residents $(n=61)$ and staff $(n=34)$ of a long-term care home in Ontario

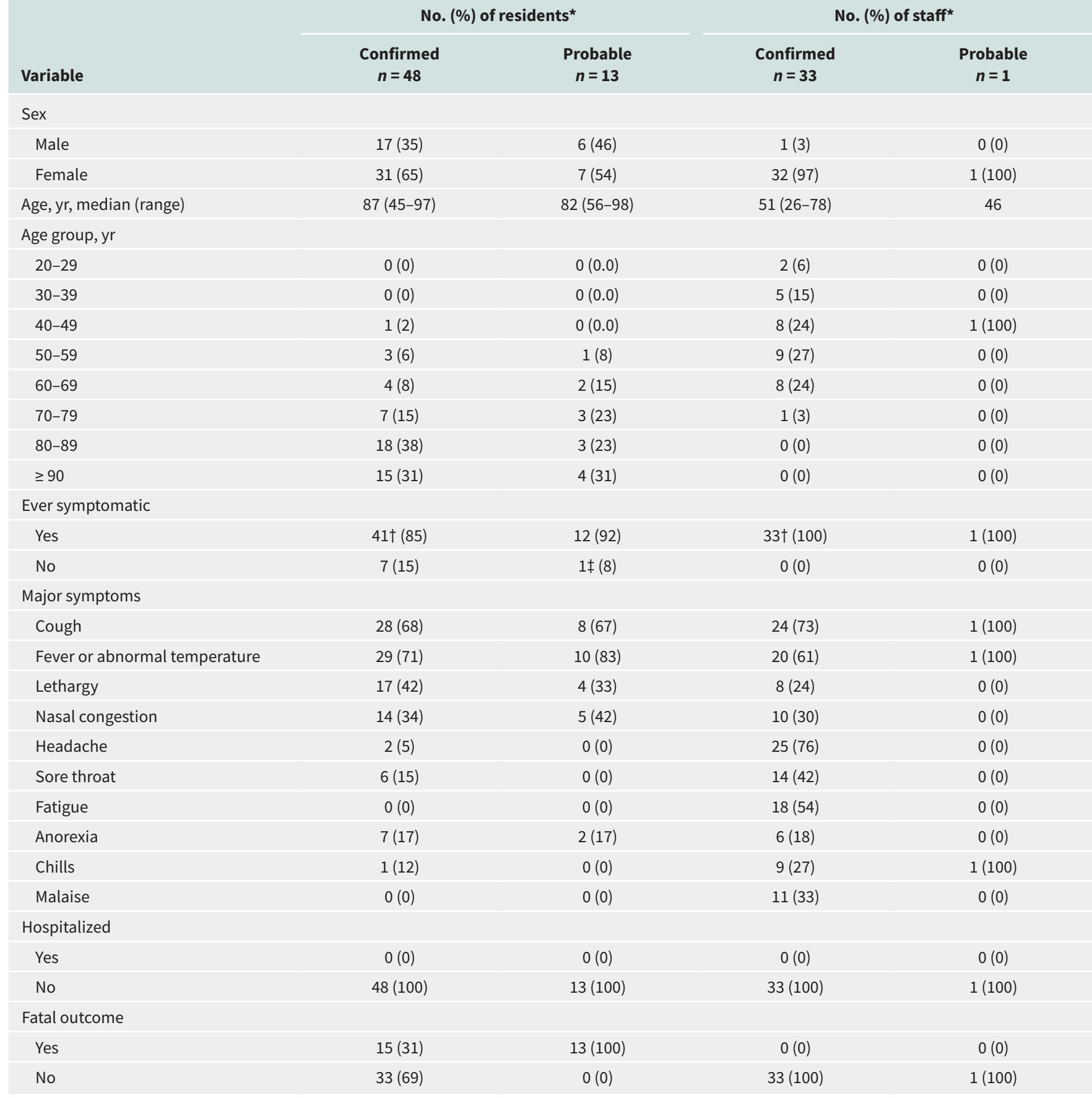

*Unless indicated otherwise.

†Includes 3 residents and 1 staff who were asymptomatic at the time of original specimen collection that tested positive, but who subsequently developed symptoms. fDeceased and was never tested; was determined to be a probable case based on assessment by the public health unit.

compared with the Wuhan-Hu-1 reference strain, and all belonged to the B.1 lineage. Phylogenetic analysis showed that all sequenced specimens of SARS-CoV-2 were highly genetically similar, with just $0-4$ SNPs separating any 2 sequences (Figure 2$)$, and $60.7 \%(n=17)$ forming a single group, with 0 SNP differences between them.
Epidemiological data showed that a staff member (ONPHL-20-00235 in Figure 2) had recently returned from international travel before the identification of the outbreak, raising suspicion that this individual introduced the virus into the LTC home, given that most cases in the province were related to travel at that time. However, the individual returned to work 
Table 2: Progression of SARS-CoV-2 spread among residents $(n=65)$ in a long-term care home based on room occupancy (March and April 2020)*

No. of residents with confirmed and probable COVID-19

(cumulative number of rooms affected)

\begin{tabular}{lcc} 
Room occupancy & $\begin{array}{c}\text { Mar. 12-17, before } \\
\text { outbreak } \\
\text { declaration } \\
\boldsymbol{n}=\mathbf{5}\end{array}$ & $\begin{array}{c}\text { Mar. 18-21, } \\
\text { outbreak peak } \\
\boldsymbol{n}=\mathbf{3 0}\end{array}$ \\
\hline Single & $1(1)$ & $2(3)$ \\
\hline Double & $1(1)$ & $16(12)$ \\
Quadruple & $3(2)$ & $12(7)$
\end{tabular}

Mar. 22-Apr. 5,
before
asymptomatic
testing
$n=16$

$3(6)$

6 (13)

7 (8)

Apr. 6-10, after
asymptomatic
testing
$n=10$

$1(7)$

$2(13)$

$7(8)$
No. of residents who never met case definition $n=4$

0

1

3

${ }^{\star}$ Room assignments that changed because of cohorting from Apr. 1 to Apr. 3 are not reflected.

when there were already symptomatic cases in the home and before the onset of their own symptoms. The SARS-CoV-2 genome sequence isolated from this individual was identical to those isolated from outbreak cases with earlier symptom onset. Thus, it is most likely that this individual was infected locally, rather than through travel. In contrast, the sequence of specimen ON-PHL-20-00238, collected from a staff case in the third week of the outbreak (based on their earliest symptom onset), appears genetically distinct, with 3 SNPs to the nearest sequence. As this SNP difference exceeds the expected number of mutations for SARS-CoV-2, this likely represents a separate introduction into the facility. ${ }^{14} \mathrm{~A}$ specimen from the last staff case was not available for analysis.

\section{Interpretation}

Outbreaks of SARS-CoV-2 in LTC during the pandemic have been severe. ${ }^{15}$ We report on one of the first LTC outbreaks in Ontario, when there were only a few cases in the region and 274 cases in the province, that resulted in one of the highest attack rates observed to date. ${ }^{1,16}$ The epidemic curve, distribution of cases in the home, phylogenetic analysis and assessment of IPAC measures suggest that introduction of virus, which occurred before the provincial implementation of specific measures for COVID-19 prevention in LTC, led to rapid spread among staff, residents and visitors. However, subsequent control measures likely limited ongoing staff transmission, with no staff cases observed among the 26 new staff introduced during the outbreak. Lessons learned from this early experience support currently recommended prevention measures and early aggressive management of cases and outbreaks in LTC.

In Ontario, initial recommendations for active screening of all LTC staff, residents and visitors for COVID-19 did not include atypical symptoms, common among older adults, or loss of smell or taste that were identified as common symptoms later in the pandemic. ${ }^{11}$, Symptoms that began in 9 staff cases before the outbreak was declared may have been missed because of a lack of awareness of the full spectrum of COVID-19 symptoms at that time. Additionally, pre-existing staffing shortages exacerbated by the outbreak may have contributed to presenteeism among symptomatic staff and spread. Universal masking of staff was not implemented until Mar. 20. Provincial measures now require that staff are excluded from work if displaying any symptoms of COVID-19 and universal masking for source control. ${ }^{7}$ Ongoing shortfalls in LTC human health resources continue to contribute to outbreak management challenges. ${ }^{17}$

The number of staff and resident cases in the LTC home before the outbreak was declared, and the relatedness of their genomic sequences, suggests an initial single introduction of SARS-CoV-2 or concurrent introduction by multiple people infected with highly related strains, that led to rapid spread. The distribution of resident cases throughout the home early in the outbreak suggests that staff may have transmitted to residents or that resident-resident transmission occurred in communal areas. Resident-resident spread in rooms with double and quadruple occupancy or in communal areas, and the overall design of the home, may have contributed to the higher attack rate, as shown in analyses of crowdedness and design in LTC homes. ${ }^{18,19}$ Use of the provincial case definition of suspect respiratory outbreaks (i.e., 2 or more cases of acute respiratory infection without laboratory confirmation) on Mar. 17 may have delayed identification of the outbreak when the initial resident developed symptoms on Mar. 12. ${ }^{9}$ Provincial COVID-19 outbreak guidance for LTC was subsequently updated to consider a single case in a resident or staff as evidence of an outbreak. ${ }^{20}$

Initial testing of all residents and staff when a SARS-CoV-2 outbreak is declared in LTC, as is now provincial guidance, may have led to earlier and more fulsome identification of cases within this outbreak. ${ }^{11}$ Repeat testing during the outbreak and postmortem testing are also currently recommended for identification of cases, but was not present during the early stages of this outbreak. Early identification of asymptomatic and presymptomatic cases assists with identifying outbreak spread and implementing cohorting measures. One published report of universal testing of LTC residents in the United States found $39.6 \%$ additional, asymptomatic cases along with symptomatic cases. ${ }^{21}$ A study from Michigan concluded that repeat testing every 3-7 days over the course of an LTC outbreak may be necessary to find additional asymptomatic cases while the outbreak is ongoing. ${ }^{22}$ Our identification of higher cycle threshold 


\begin{tabular}{|l|}
\hline Case type \\
O Staff \\
O Resident \\
Symptom onset \\
Week 1, Mar. 9-15 \\
Week 2, Mar. 16-22 \\
Week 3, Mar. 23-29 \\
Week 4, Mar 30-Apr. 5 \\
\hline
\end{tabular}

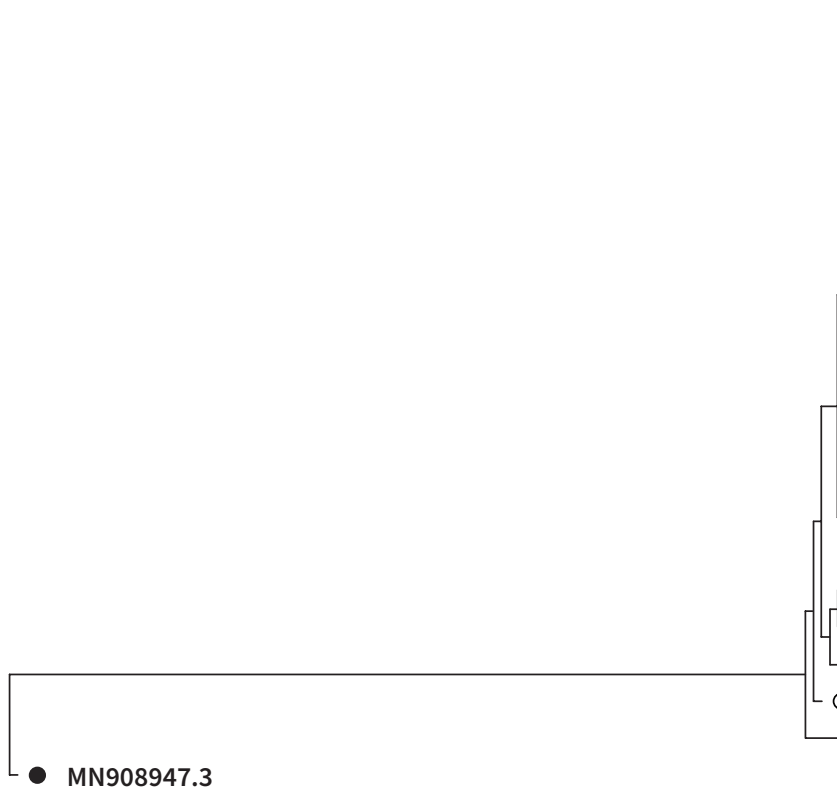

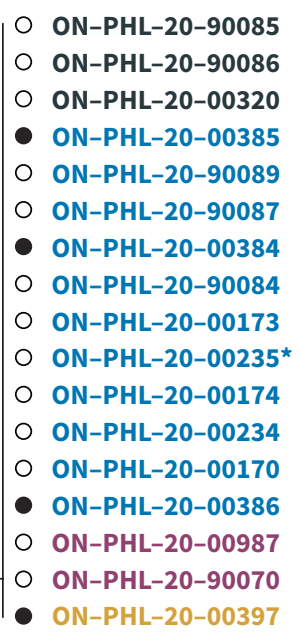

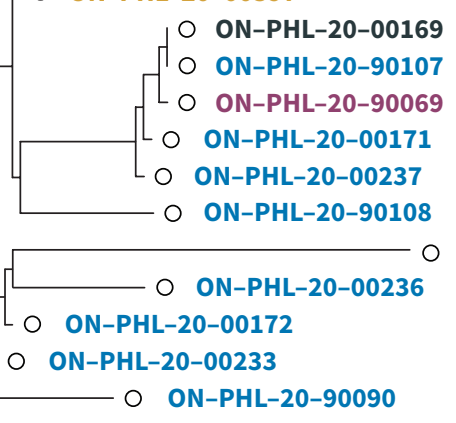

ON-PHL-20-00238

$3 e-05$

Figure 2: Maximum likelihood tree showing the phylogenetic relationship between 28 SARS-CoV-2 genomes from specimens collected from staff and residents in March and April 2020. The tree was rooted using the reference genome, Wuhan-Hu-1 (accession MN908947.3). Note: Phylogenetic analysis depicts the evolutionary relationship between sequences, with horizontal branch lengths corresponding to genetic distance (nucleotide substitutions per site). ON-PHL-2000238 is genetically distinct, with 3 single nucleotide polymorphisms to the nearest sequence. * ${ }^{*}$ taff member had travelled internationally before the outbreak.

values from specimens collected more than 14 days from symptom onset and from specimens of asymptomatic cases suggests that early testing of symptomatic cases and repeat testing of people who are asymptomatic may prevent falsenegative results from late or missed testing. Serology testing was not available to assess for the possibility of late falsenegative results among residents who remained asymptomatic and tested negative later in the outbreak, who may have been infected early in the outbreak and cleared by the time they were tested. No other reasons were identified as to why $4(6 \%)$ residents continued to test negative for SARS-CoV-2, given their exposure and susceptibility.

Strengths of our study include the detailed collaboration with the health unit and the LTC home, which allowed a far greater depth of analysis of the initial days of the outbreak and the challenges experienced early in the pandemic in Canada than has been reported in other case reports. This detailed analysis provided additional insights into the factors that likely contributed to spread within the LTC home. Our report also showed the effectiveness of outbreak measures implemented to prevent infection among staff; no subsequent infections were identified among new staff introduced into the facility after outbreak measures were implemented, even though there were still infectious cases present. Our analysis of cycle threshold values also provides new insights as to why cases in the first wave of the pandemic were likely missed when there was late testing of people who were symptomatic and no routine testing of those who were asymptomatic. Large-scale whole genome sequencing of SARS-CoV-2 has made it possible to identify discrete lineages as they arise and disseminate globally, and to distinguish between travel-related infections, local transmission and multiple outbreak introductions, as we identified in this analysis. ${ }^{23-25}$ Few outbreak reports have included a phylogenetic analysis with their epidemiological analysis as our report did, providing additional insights into transmission in the LTC home, particularly at the start of the pandemic, when there were few cases overall in Ontario. 


\section{Limitations}

One limitation of our genomic investigation is that only a relatively small proportion $(<30 \%)$ of specimens from the total cases were sequenced, as some viral samples were no longer available or had insufficient viral load that did not allow for adequate genome coverage. ${ }^{26,27}$

Other study limitations include incomplete information regarding visitors to the home to link any additional visitor cases, and limited information on staff-staff, staff-visitor, visitorresident or resident interactions outside the LTC home that may have contributed to initial virus introductions. Additionally, staff and visitor cases may be underrepresented if never tested or tested late in the outbreak.

\section{Conclusion}

Lessons learned from this early and severe outbreak of SARSCoV-2 in an LTC home have helped to inform the current provincial prevention and outbreak measures, specifically, the need for early identification of symptoms, cases and outbreaks, and for implementation of additional testing and prevention measures. Active screening and universal masking for staff are needed to prevent the introduction and asymptomatic spread of SARSCoV-2 from staff. Mass testing of residents and staff in an outbreak allows for early identification of cases to facilitate outbreak measures. Ensuring sufficient staffing and personal protective equipment is necessary for appropriate care delivery, protection for staff and prevention of transmission in the home. Despite the effectiveness of these measures to prevent transmission after outbreaks arise, the prevention of LTC outbreaks requires low levels of community transmission and improvements to LTC infrastructure, density and staffing.

\section{References}

1. COVID-19 data and surveillance. Toronto: Ontario Agency for Health Protection and Promotion (Public Health Ontario); 2020. Available: https://www. publichealthontario.ca/en/data-and-analysis/infectious-disease/covid-19-data -surveillance (accessed 2020 July 4).

2. Coronavirus disease 2019 (COVID-19): epidemiology update. Ottawa: Government of Canada; 2020. Available: https://health-infobase.canada.ca/covid-19/ epidemiological-summary-covid-19-cases.html (accessed 2020 July 4).

3. Coronavirus disease (COVID-19) weekly epidemiological update and weekly operational update. Geneva: World Health Organization; 2020. Available: https://www.who.int/emergencies/diseases/novel-coronavirus-2019/situation -reports/ (accessed 2020 July 4).

4. Volling C, Popalyar A, Defalco K, et al.; Public Health Agency of Canada; National Advisory Committee on Infection Prevention and Control (NAC-IPC). Infection prevention and control for COVID-19: interim guidance for long term care homes. Ottawa: Government of Canada; 2020. Available: https://www. canada.ca/en/public-health/services/diseases/2019-novel-coronavirus-infection/ prevent-control-covid-19-long-term-care-homes.html\#a18 (accessed 2020 July 4).

5. Weekly epidemiologic summary: Focus on January 31, 2021 - February 6, 2021. Toronto: Ontario Agency for Health Protection and Promotion (Public Health Ontario); 2021. Available: https://www.publichealthontario.ca/-/media/docu ments/ncov/epi/covid-19-weekly-epi-summary-report.pdf?la=en (accessed 2021 Feb. 13).

6. Pollard B. COVID-19 updates [memorandum to: long-term care homes sector]. Toronto: Ministry of Long-Term Care; 2020 Mar. 9. Available: https://www.oha. com/Bulletins/MLTC\%20ADM\%20Memo-COVID19\%20Updates\%20March\%20 09\%202020.pdf (accessed 2020 July 4).

7. COVID-19: orders, directives, memorandums and other resources. Toronto: Ontario Ministry of Health and Long-Term Care; 2020. Available: http://www. health.gov.on.ca/en/pro/programs/publichealth/coronavirus/dir_mem_res. aspx (accessed 2020 July 4).
8. Enhanced epidemiologic summary: COVID-19 in long-term care home residents in Ontario - January 15, 2020 to June 1, 2020. Toronto: Ontario Agency for Health Protection and Promotion (Public Health Ontario); 2021. Available: https://www.publichealthontario.ca/-/media/documents/ncov/epi/2020/06/ covid-19-epi-ltch-residents.pdf?la=en (accessed 2020 July 4).

9. Appendix A: disease-specific chapters: respiratory infection outbreaks in institutions and public hospitals. Toronto: Ministry of Health and Long-Term Care; 2019. Available: http://www.health.gov.on.ca/en/pro/programs/publichealth/ oph_standards/docs/respiratory_outbreaks_chapter.pdf (accessed 2020 July 4).

10. Ontario COVID-19 data tool. Toronto: Ontario Agency for Health Protection and Promotion (Public Health Ontario); 2020. Available: https://www. publichealthontario.ca/en/data-and-analysis/infectious-disease/covid-19-data -surveillance/covid-19-data-tool (accessed 2020 July 4).

11. COVID-19: guidance for the health sector. Toronto: Ontario Ministry of Health and Long-Term Care; 2020. Available: http://www.health.gov.on.ca/en/pro/ programs/publichealth/coronavirus/2019_guidance.aspx\#case (accessed 2020 July 22).

12. Corman VM, Kaiser M, Molenkamp R, et al. Detection of 2019 novel coronavirus (2019-nCoV) by real-time RT-PCR. Euro Surveill 2020;25:2000045. doi: 10.2807/1560-7917.ES.2020.25.3.2000045.

13. Control of respiratory infection outbreaks in long-term care homes, 2018. Toronto: Ontario Ministry of Health and Long-Term Care; 2018. Available: http://www.health.gov.on.ca/en/pro/programs/publichealth/oph_standards/ docs/reference/RESP_Infectn_ctrl_guide_LTC_2018_en.pdf (accessed 2020 July 22).

14. Rambaut A. Phylogenetic analysis of $\mathrm{nCoV}-2019$ genomes. virological.org 2020 Mar. 06. Available: https://virological.org/t/phylodynamic-analysis -176-genomes-6-mar-2020/356 (accessed 2020 Aug. 24).

15. Fisman DN, Bogoch I, Lapointe-Shaw L, et al. Risk factors associated with mortality among residents with coronavirus disease 2019 (COVID-19) in long-term care facilities in Ontario, Canada. JAMA Netw Open 2020;3:e2015957. doi: 10.1001/jamanetworkopen.2020.15957.

16. Salcher-Konrad M, Jhass A, Naci H, et al. COVID-19 related mortality and spread of disease in long-term care: a living systematic review of emerging evidence. medRxiv 2020 Aug. 1. Available: https://doi.org/10.1101/2020.06.09.20125237 (accessed 2020 July 4).

17. Liu M, Maxwell CJ, Armstrong P, et al. COVID-19 in long-term care homes in Ontario and British Columbia. CMAJ 2020;192:E1540-6.

18. Brown KA, Jones A, Daneman N, et al. Association between nursing home crowding and COVID-19 infection and mortality in Ontario, Canada. JAMA Intern Med 2021;181:229-36. Available: https://jamanetwork.com/journals/ jamainternalmedicine/fullarticle/2772335 (accessed 2021 Feb. 13).

19. Stall NM, Jones A, Brown KA, et al. For-profit long-term care homes and the risk of COVID-19 outbreaks and resident deaths. CMAJ 2020;192:E946-55.

20. COVID-19 outbreak guidance for long-term care homes (LTCH): version 2 April 15, 2020. Toronto: Ontario Ministry of Health; 2020. Available: http:// www.health.gov.on.ca/en/pro/programs/publichealth/coronavirus/docs/LTCH_ outbreak_guidance.pdf (accessed 2020 July 24).

21. Bigelow B, Tang O, Barshick B, et al. Outcomes of universal COVID-19 testing following detection of incident cases in 11 long-term care facilities. JAMA Intern Med 2021;181:127-9.

22. Sanchez GV, Biedron C, Fink LR, et al. Initial and repeated point prevalence surveys to inform SARS-CoV-2 infection prevention in 26 skilled nursing facilities Detroit, Michigan, March-May 2020. MMWR Morb Mortal Wkly Rep 2020;69:882-6. doi: 10.15585/mmwr.mm6927e1.

23. Rambaut A, Holmes EC, O'Toole Á, et al. A dynamic nomenclature proposal for SARS-CoV-2 lineages to assist genomic epidemiology. Nat Microbiol 2020; 5:1403-7.

24. Eden JS, Rockett R, Carter I, et al. An emergent clade of SARS-CoV-2 linked to returned travellers from Iran. Virus Evol 2020;6:veaa027. doi: 10.1093/ve/ veaa027.

25. Geoghegan JL, Ren X, Storey M, et al. Genomic epidemiology reveals transmission patterns and dynamics of SARS-CoV-2 in Aotearoa New Zealand. medRxiv 2020 Aug. 20. Available: https://doi.org/10.1101/2020.08.05.20168930 (accessed 2020 Aug. 23).

26. Seemann T, Lane CR, Sherry NL, et al. Tracking the COVID-19 pandemic in Australia using genomics. Nat Commun 2020;11:4376. doi: 10.1038/ s41467-020-18314-x.

27. Lemieux J. Introduction and spread of SARS-CoV-2 in the greater Boston area. virological.org 2020 June 4. Available: https://virological.org/t/introduction -and-spread-of-sars-cov-2-in-the-greater-boston-area/503 (accessed 2020 Aug. 23). 
Competing interests: None declared.

This article has been peer reviewed.

Affiliations: Public Health Ontario (Murti, Saunders, Guthrie, Eshaghi, Zittermann, Teatero, Fittipaldi, Rilkoff, Gubbay, Garber, Callery); University of Toronto (Murti, Fittipaldi, Gubbay, Garber), Toronto, Ont.; Haliburton, Kawartha, Pine Ridge District Health Unit (Goetz, Sunil, Holt, Noseworthy), Port Hope, Ont.

Contributors: Michelle Murti conceived and designed the work. Monika Goetz, Andrea Saunders, Vidya Sunil, Jennifer Guthrie, AliReza Eshaghi, Sandra Zittermann, Sarah Teatero, Nahuel Fittipaldi, Heather Rilkoff, Jonathan Gubbay, Gary Garber, Sandra Callery, Anne Marie Holt, A. Lynn Noseworthy acquired, analyzed and interpreted data. Michelle Murti drafted the manuscript, which all authors critically revised for intellectual content. All authors approved the final version to be published and agreed to be accountable for all aspects of the work.

Funding: Support for this work was provided by the organizational mandates of Public
Health Ontario and the Haliburton, Kawartha, Pine Ridge District Health Unit. Genome data acquisition and analysis was supported in part by a grant from Genome Canada to Nahual Fittipaldi under the umbrella of the Canadian COVID Genomics Network (CanCoGeN). CanCoGeN had no role in the writing of this manuscript.

Content licence: This is an Open Access article distributed in accordance with the terms of the Creative Commons Attribution (CC BY-NC-ND 4.0) licence, which permits use, distribution and reproduction in any medium, provided that the original publication is properly cited, the use is noncommercial (i.e., research or educational use), and no modifications or adaptations are made. See: https://creativecommons. org/licenses/by-nc-nd/4.0/

Data sharing: Public Health Ontario $(\mathrm{PHO})$ and Haliburton, Kawartha, Pine Ridge District Health Unit (HKPRDHU) cannot disclose the underlying data as doing so would compromise individual privacy contrary to $\mathrm{PHO}$ and HKPRDHU's ethical and legal obligations. Restricted access to the data may be available under conditions prescribed by the Ontario Personal
Health Information Protection Act, 2004, the Ontario Freedom of Information and Protection of Privacy Act, the Tri-Council Policy Statement: Ethical Conduct for Research Involving Humans, and $\mathrm{PHO}$ privacy and ethics policies. Data are available for researchers who meet PHO's criteria for access to confidential data. Information about PHO's data access request process is available at www.publichealthontario.ca/en/ data-and-analysis/using-data/data-requests.

Acknowledgements: The authors acknowledge the contributions to the phylogenetic analysis from Brenda Stanghini, Yao Chen, Aimin Li and the Public Health Ontario Laboratory Biocomputing Centre staff, as well as Jared Simpson at the Ontario Institute for Cancer Research. The authors also thank Shelly Griffin, Mary Carr, Julie Parcells and other staff at the long-term care home, as well as staff at Haliburton, Kawartha, Pine Ridge District Health Unit.

Accepted: Apr. 8, 2021

Correspondence to: Michelle Murti, Michelle. murti@oahpp.ca 